\title{
Litchi und Rambutan, zwei Seifenbaumgewächse mit ungewöhnlichen Früchten
}

\author{
VEIT M. DÖRKEN
}

\begin{abstract}
Lychee (Litchi chinensis) and rambutan (Nephelium lappaceum) are evergreen tropical trees. Their delicious fruits are edible. Morphology, biology and use of these species are outlined.
\end{abstract}

\section{Zusammenfassung}

Litchi (Litchi chinensis) und Rambutan (Nephelium lappaceum) sind immergrüne tropische Bäume. Ihre wohlschmeckenden Früchte sind essbar. Morphologie, Biologie und Verwendung werden vorgestellt.

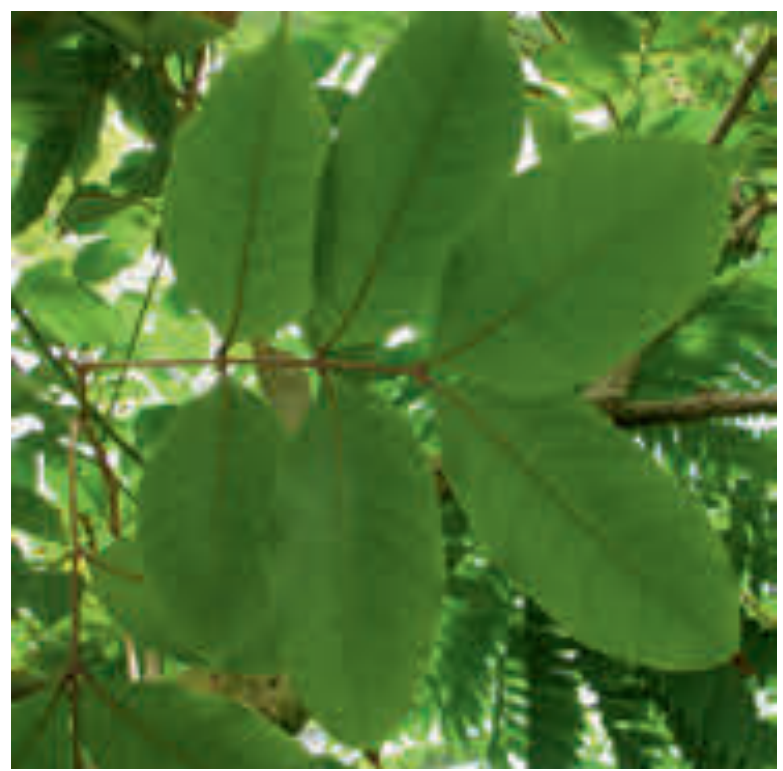

\section{Einleitung}

In den letzten Jahren ist bei uns eine Zunahme von exotischen Früchten zu verzeichnen, die besonders in den Wintermonaten im deutschen Lebensmittelhandel angeboten werden (vgl. Hetzel \& Jagel 2011). Viele der exotischen Früchte sind zwar als Konservenobst schon lange erhältlich, konnten aber erst nach der Verbesserung von Transport- und Lagerungsbedingungen auch als Frischobst angeboten werden. Aufgrund ihrer eigentümlichen, fast schon künstlich anmutenden Oberflächen sind Litchis (Litchi chinensis, auch Lychee, Litschipflaume oder Chinesische Haselnuss genannt) und Rambutan (Nephelium lappaceum, Falsche Litschi) wohl die auffälligsten und ungewöhnlichsten Früchte, die die Frischobsttheken in den letzten Jahren erobert haben. Sie werden vor allem ab der Adventszeit bis in den Januar hinein angeboten. Litchi und Rambutan zählen in Ost-Asien zum beliebtesten Obst. Hier wird die Litchi sogar zu einer der feinsten Früchte der Welt gezählt.

\section{Systematik}

Die beiden nahe verwandten Gattungen Litchi (Litchi) und Rambutan (Nephelium) gehören zu den Seifenbaumgewächsen (Sapindaceae). Zu dieser Pflanzenfamilie im weiteren Sinne werden heute auch die in Europa heimischen Gattungen Acer (Ahorn, früher Aceraceae) und Aesculus (Rosskastanie, früher Hippocastanaceae) gestellt. Litchi und Rambutan sind artenarme Gattungen, wobei Litchi mit nur einer Art monotypisch ist. Allerdings werden von Litchi drei Unterarten unterscheiden: chinensis (China), philippinensis (Philippinen) und javensis (Java, Indonesien).

Litchi chinensis wurde ehemals unter Nephelium chinensis geführt, bevor sie in der eigenständigen Gattung Litchi ausgegliedert wurde. Die Gattung Nephelium ist mit 22 Arten artenreicher (MabBerley 2008).

\section{Verbreitung}

Litchi und Rambutan waren beide ursprünglich nur im ostasiatischen Raum verbreitet, bevor sie als Obstgehölze in adäquaten Klimaten auch auf anderen Kontinenten angebaut wurden. Litchi stammt ursprünglich aus Süd-China, wo sie schon seit über 4000 Jahren kultiviert wird. Rambutan hat seine natürliche Heimat in den feuchttropischen Regionen des 
malaysischen Archipels, der Philippinen und Indiens. Litchi gelangte im ausgehenden 17. Jh. über Burma nach Indien und von dort aus erst im 19 Jh. auch in andere Erdteile. Heutzutage befinden sich die weltgrößten Litchi-Plantagen fernab des Naturstandortes (KRATOCHVIL 1995). Wichtige Anbaugebiete sind China, Taiwan, Thailand, Indien, Südafrika, Madagaskar, Mauritius und Australien. Rambutan wird rund um den Äquator in feucht-tropischen Gebieten angebaut. Wichtigster Produzent ist Thailand.

\section{Morphologie}

Alle Arten sind kleine immergrüne Bäume mit wechselständig stehenden, gefiederten Blättern. Die kleinen Blüten stehen in reichblütigen Infloreszenzen. Bei Litchi gibt es auf ein und derselben Pflanze drei verschiedene Blütentypen: männliche, weibliche und zwittrige (die letzteren sind aber funktionell ebenfalls weiblich, die Staubblätter sind hier nicht funktionstüchtig). Bei Rambutan sind sie auf verschiedene Pflanzen verteilt, so dass es weibliche und männliche Pflanzen gibt.

Die Fruchtschale der Litchi ist aus kleinen fünf- bis sechseckigen Feldern zusammengesetzt, bei Rambutan ist jedes dieser Felder mit einem langen, spitzen und borstigen Auswuchs versehen. Darauf nimmt auch die volkstümliche Bezeichnung im Ursprungsland des Rambutans Bezug (rambut $=$ Haar).

Der Fruchtknoten besteht aus zwei Fruchtblättern, die jeweils nur eine Samenanlage enthalten. Normalerweise entwickelt sich nur ein Fruchtblatt weiter zur Frucht, selten aber auch beide Fruchtblätter. Dann entstehen so genannte Zwillingspflaumen, die man in Ost-Asien als Glückssymbole betrachtet (Lieberei \& ReisDORFF 2007).

Abb. 1 (Seite 128): Blatt von Litchi chinensis.

Abb. 2 (oben): Reife Frucht der Litchi.

Abb. 3 (Mitte): Halbierte Litchi.

Abb. 4 (unten): Blühender Zweig von Nephelium coriaceum, einer Verwandten von Rambutan.
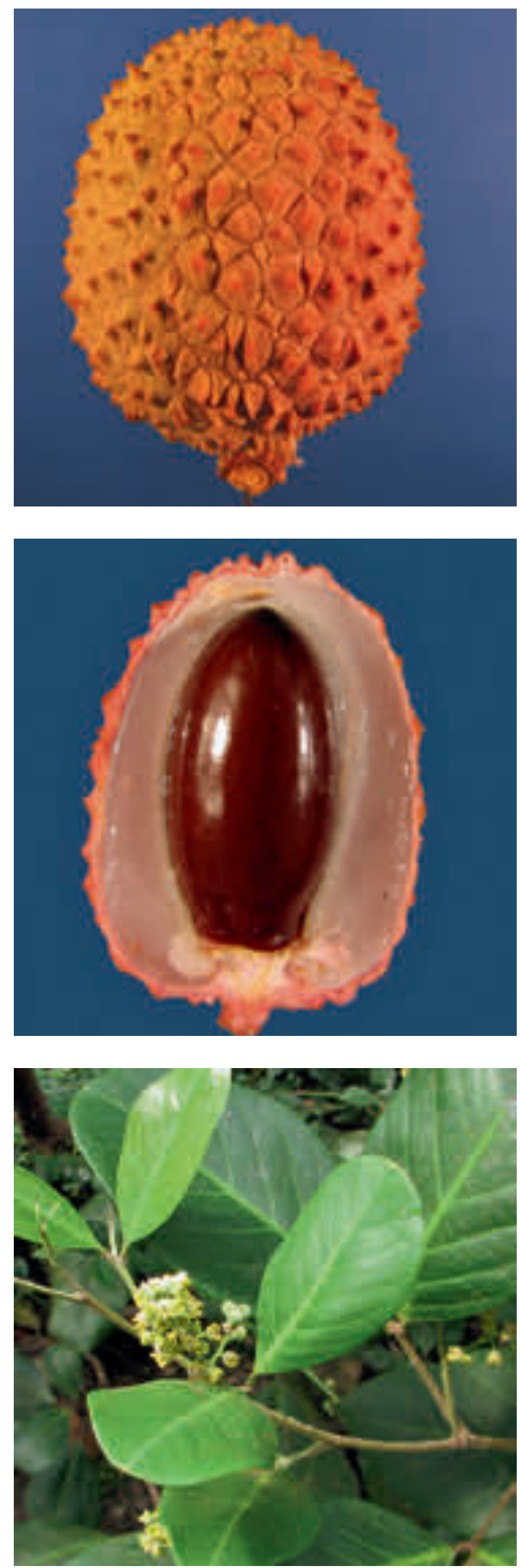

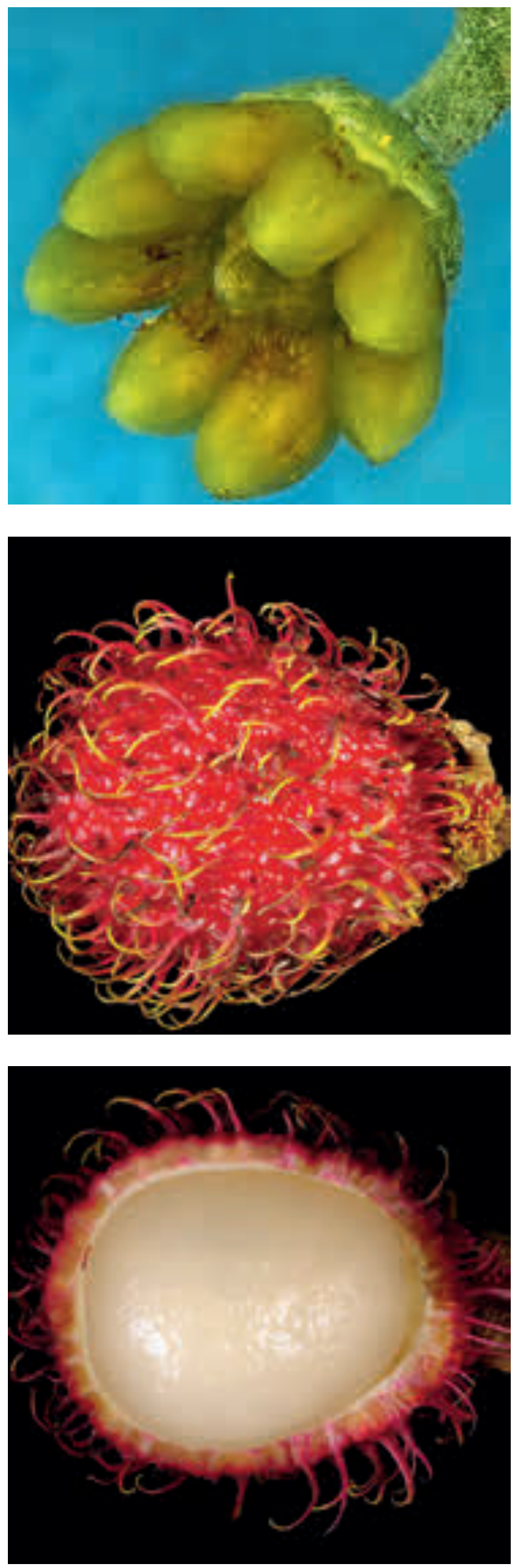

Die Früchte sind einsamige Nüsse, auch wenn sie nicht nach Nüssen aussehen. Ihre gesamte Fruchtwand (Schale) ist aber, wie für Nüsse typisch, trocken und holzig bzw. ledrig. Das, was man als Fruchtfleisch verzehrt, ist keine Bildung der Fruchtwand. Die weiße, fleischige, süßlich schmeckende Schicht zwischen der Schale und dem großen braunen, länglichen Samen ist nicht mit dem weichen Fruchtanteil von Beeren oder Steinfrüchten vergleichbar. Vielmehr handelt es sich um einen Auswuchs des Trägers, an dem der Samen sitzt (Funiculus). Von diesem Samenmantel (Arillus) wird der Same umgeben. Samenmäntel gibt es auch bei heimischen Arten. So ist die rote, fleischige und essbare Hülle, die den Samen der Eibe umgibt, ebenfalls ein Arillus.

$100 \mathrm{~g}$ frische Litchi enthalten u.a. 16,53g Kohlenhydrate, $44 \mathrm{~g}$ Fette und 15,23 g Zucker, außerdem die Spurenelemente Calcium, Magnesium, Kalium, Phosphor und Vitamin C. Mit einem Gesamtanteil von etwa $80 \%$ am gesamten Säuregehalt stellt Apfelsäure die Hauptsäure dar (Herrmann 1983, Lieberei \& ReisDORFF 2007).

\section{Köstliches Obst}

Litchi und Rambutan sind nicht nur für Menschen, sondern am Naturstandort auch für Affen eine Delikatesse. Nach dem Fressen werden die Samen ausgespuckt oder weggeworfen und somit ausgebreitet.

Von Litchi gibt es zahlreiche großfrüchtige Sorten wie z. B. 'Mauritius', 'Kwai Mi', 'Hak Pi' oder auch 'Brewster' (Franke 1994). Für den Transport vorgesehene Litchis werden unreif geerntet und reifen später nach. Ein großes Pro-

Abb. 5 (oben): Blüte von Nephelium coriaceum.

Abb. 6 (Mitte): Reife Rambutan-Frucht.

Abb. 7 (unten): Geöffnete Rambutan-Frucht mit weißem Arillus.

Abb. 8 (Seite 131 oben): Freigelegter Same in einer geöffneten Rambutan-Frucht.

Abb. 9 (Seite 131 unten): Litchi-Früchte auf einem Marktstand in Frankfurt. 
blem ist hierbei, dass Litchis und auch Rambutan nur kurzzeitig lagerungsfähig sind, weil sie rasch zu faulen beginnen. Dabei ist Rambutan noch empfindlicher als Litchi, ein Grund dafür, warum Rambutan bei uns nicht in so großen Mengen wie Litchi angeboten wird. Die Schalen nicht gekühlter Litchis verfärben sich innerhalb von nur wenigen Tagen braun, meist einhergehend mit einer beginnenden Fäulnisbildung. Diese wird durch Schimmelpilze wie z. B. Aspergillus, Botryodiplodia, Colletotrichum, Fusarium oder Penicillium verursacht. Hohe Luftfeuchte und Temperaturen zwischen $0{ }^{\circ} \mathrm{C}$ und $1{ }^{\circ} \mathrm{C}$ sollen die Haltbarkeit von Litchis auf bis zu 30 Tage erhöhen (Franke 1994). Bei HerrMANN (1983) wird eine Temperatur von $1-2^{\circ} \mathrm{C}$ empfohlen, um die Früchte bis zu 5 Wochen lang lagern zu können.

\section{Weitere Verwendungen}

Litchi und Rambutan werden überwiegend frisch als Obst gegessen, sind aber bestens auch zum Einkochen und als Konserve geeignet. Aus dem Arillus werden Marmeladen, Gelees und Desserts angefertigt. Litchi-Kompotte eignen sich hervorragend als Beigabe zu Fisch- und Fleischgerichten. Wie Weintrauben getrocknet, liefern Litchi ein köstliches Trockenobst, wobei - wie auch beim Einkochen - viel des süßen Aromas eingebüßt wird. Getrocknete Litchis werden auch zum Aromatisieren von Tees, zur Herstellung von Wein und Likör oder zum Verfeinern von Cocktails genutzt. Nur kurz angekocht werden die Arilli von Litchi in China zu Reis gereicht. Die fettreichen (40\%) Samen von Rambutan sind im rohen, unbehandelten $\mathrm{Zu}$ stand schwach giftig, können jedoch nach dem Rösten verzehrt werden. Litchi-Samen sind hingegen aufgrund des hohen Gehaltes an Cyclopropanfettsäuren ungenießbar, werden jedoch technisch genutzt. Ein Brei aus gemahlenen Litchi-Samen wird in der Volksmedizin zur Heilung von Erkrankungen der Haut sowie zur Wundheilung eingesetzt. Außerdem wirken sie sich lindernd auf Verdauungsbeschwerden aus (Herrmann 1983, Kratochvil 1995, Novak \& Schulz 2009) ...
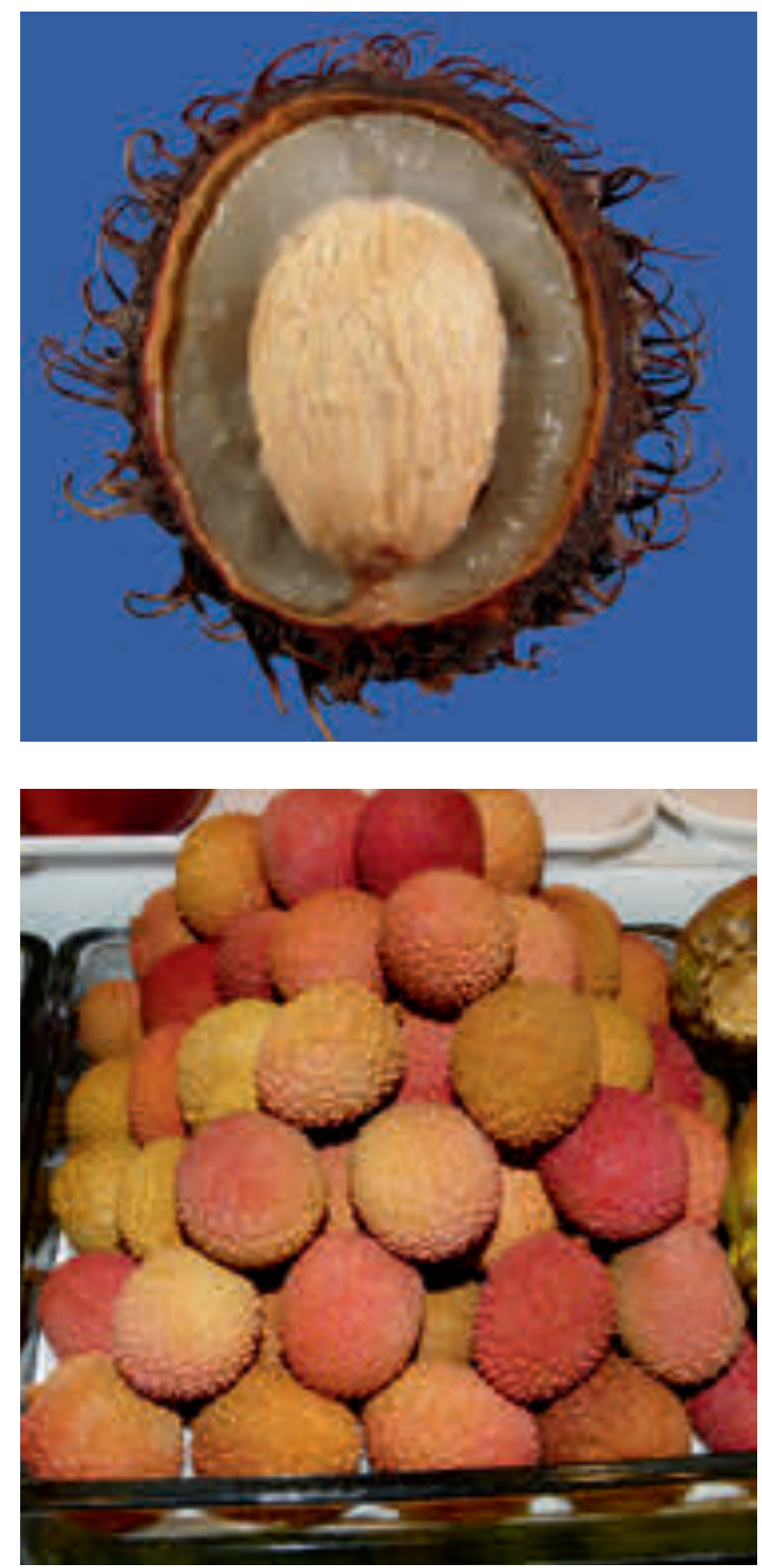

\section{Literatur}

Herrmann, K. 1983: Exotische Lebensmittel. - Berlin, Heidelberg, New York.

Hetzel, I. \& Jagel, A. 2011: Diospyros kaki- Kaki, Kakipflaume (Ebenaceae). - Jahrb. Bochumer Bot. Ver. 2: 194-198.

Franke, G. 1994: Nutzpflanzen der Tropen und Subtropen, Bd. 2: Spezieller Pflanzenbau. - Stuttgart. Kratochvil, H. 1995: Lexikon exotischer Früchte. Wien.

Lieberei, R. \& Reisdorff, C. 2007: Nutzpflanzenkunde, 7. Aufl. - Stuttgart.

Mabberley, D. J. 2008: Mabberley's plant book, 3. Aufl. - Cambridge.

Novak, B. \& Schulz, B. 2009: Taschenlexikon tropischer Nutzpflanzen und ihrer Früchte. - Wiebelsheim. 